University of New Orleans

ScholarWorks@UNO

4-2004

\title{
A reoptimization of the five-site water potential (TIP5P) for use with Ewald sums
}

Steven W. Rick

University of New Orleans, srick@uno.edu

Follow this and additional works at: https://scholarworks.uno.edu/chem_facpubs

Part of the Chemistry Commons

\section{Recommended Citation}

Steven W. Rick. 2004. "A reoptimization of the five-site water potential (TIP5P) for use with Ewald sums." Journal of Chemical Physics 120 (13): 6085-6093.

This Article is brought to you for free and open access by the Department of Chemistry at ScholarWorks@UNO. It has been accepted for inclusion in Chemistry Faculty Publications by an authorized administrator of ScholarWorks@UNO.For more information, please contact scholarworks@uno.edu. 


\title{
A reoptimization of the five-site water potential (TIP5P) for use with Ewald sums
}

\author{
Steven W. Rick ${ }^{\mathrm{a})}$ \\ Department of Chemistry, University of New Orleans, New Orleans, Louisiana 70148 and Chemistry \\ Department, Southern University of New Orleans, New Orleans, Louisiana 70126
}

(Received 10 November 2003; accepted 9 January 2004)

\begin{abstract}
The five-site transferable interaction potential (TIP5P) for water [M. W. Mahoney and W. L. Jorgensen, J. Chem. Phys. 112, 8910 (2000)] is most accurate at reproducing experimental data when used with a simple spherical cutoff for the long-ranged electrostatic interactions. When used with other methods for treating long-ranged interactions, the model is considerably less accurate. With small modifications, a new TIP5P-like potential can be made which is very accurate for liquid water when used with Ewald sums, a more physical and increasingly more commonly used method for treating long-ranged electrostatic interactions. The new model demonstrates a density maximum near $4{ }^{\circ} \mathrm{C}$, like the TIP5P model, and otherwise is similar to the TIP5P model for thermodynamic, dielectric, and dynamical properties of liquid water over a range of temperatures and densities. An analysis of this and other commonly used water models reveals how the quadrupole moment of a model can influence the dielectric response of liquid water. (C) 2004 American Institute of Physics.
\end{abstract} [DOI: $10.1063 / 1.1652434]$

\section{INTRODUCTION}

Water potentials are a key component in the simulation of molecular systems. The success of these models impacts not only the great many simulations which include water but can be used to assess the importance of different interaction models. A large amount of effort has gone into the development of water models, more than for any other molecule and the wealth of experimental information on bulk water can be used to judge the models. Recent studies have addressed many issues concerning the development and accuracy of models for water and aqueous solutions, including the inclusion of polarizability and the introduction of more interaction sites. ${ }^{1-3}$ One recent water model, representing an approach to improve potentials by adding off-atom interaction sites, is the TIP5P model of Mahoney and Jorgensen. ${ }^{2}$ The TIP5P model has been shown to be successful in reproducing many of the properties of liquid water, including the dielectric constant, the diffusion constant, and the density maximum near $4{ }^{\circ} \mathrm{C}$. ${ }^{2,4}$ The last feature is especially noteworthy since many water models do not successfully reproduce this important property of water, although a small number of potentials do also have a density maximum near $4{ }^{\circ} \mathrm{C}^{3,5-7}$ The success of the TIP5P model has led to its widespread use. ${ }^{4,8-17}$

Computer simulations involving periodic boundary conditions and long-ranged interactions require a method for treating the interactions beyond the central simulation cell. Common methods include simple spherical cutoff, Ewald sums, switching functions, and reaction field methods. ${ }^{18}$ The TIP5P model is parameterized to be used with the longranged interactions truncated at $9 \AA$. Problems associated with the use of sharp cutoffs and the importance of using Ewald sums has been demonstrated for the simulation of

${ }^{\text {a)} E l e c t r o n i c ~ m a i l: ~ s r i c k @ u n o . e d u ~}$ water ${ }^{19-25}$ and ionic solutions, ${ }^{26-29}$ as well as proteins and peptides. ${ }^{30-34}$ One general problem introduced by the use of cutoffs is an upward drift in the total energy of the system. ${ }^{35}$ Increasingly, the use of Ewald or particle mesh Ewald has become the standard approach and is now included in common molecular simulation packages, including $\mathrm{AMBER}^{36}$ and CHARMM. ${ }^{37}$ Due to boundary condition effects, the properties of the TIP5P model have been shown to be size dependent and change noticeably when the size of the system is changed from 512 to 216 molecules, with a change in the cutoff to $8 \AA{ }^{2}$ In addition, the properties also change when Ewald or reaction field methods are used. ${ }^{38}$ The TIP5P model apparently shows a more significant dependence on systems size that the TIP4P model. ${ }^{39,40}$ Most notably, the density, at a pressure of $1 \mathrm{~atm}$ and a temperature of $298 \mathrm{~K}$, changes from 0.999 ( 512 molecules with a $9 \AA$ cutoff) to $0.985 \mathrm{~g} / \mathrm{cm}^{3}$ (512 molecules with Ewald) and the potential energy changes from -9.867 to $-9.682 \mathrm{kcal} / \mathrm{mol}$, meaning that the addition of long-ranged interactions makes the liquid less dense and more weekly interacting. Using Ewald, the dependence on system size is much smaller. ${ }^{38}$ Other comparisons of Ewald and cutoffs with other water potentials also find an increase in the potential and a decrease in the density (or at constant volume, an increase in the pressure). ${ }^{21,24,25}$ When simulating pure water with the TIP5P model, the use of a $9 \AA$ cutoff may be the best method, but for heterogeneous systems, using potentials which have not necessarily been developed using cutoffs, the choice is not as clear. For applications in which Ewald or reaction field methods are desired, a modified potential would be useful. A modified potential would also be useful for studies of the effects of long-ranged interactions themselves on other properties (such on as the stability of $i \mathrm{ic}^{11}$ ). Presented here is a small modification to the TIP5P potential, which, when used with Ewald, essentially 
TABLE I. Parameters for the TIP5P $\mathrm{P}^{2}$ and the TIP5P-E models.

\begin{tabular}{lccc}
\hline \hline Model & $\epsilon(\mathrm{kcal} / \mathrm{mol})$ & $\sigma(\AA)$ & $q_{\mathrm{H}}(e)$ \\
\hline TIP5P & 0.160 & 3.120 & 0.241 \\
TIP5P-E & 0.178 & 3.097 & 0.241 \\
\hline \hline
\end{tabular}

reproduces the accuracy of the TIP5P potential for thermodynamic, dielectric, and dynamical properties over a range of temperatures and pressures. In addition, an analysis of the dielectric constants of several water models, together with previous integral equation results for hard spheres, ${ }^{41}$ offers an explanation for the accurate dielectric constant for the TIP5P model, despite it relatively low dipole moment.

\section{METHODS}

\section{A. Optimization procedure}

The TIP5P potential is a rigid, five-site model for water, with charges on the two hydrogen sites, as well as two interaction sites (denoted as M-sites) in lone-pair type positions. The oxygen site interacts only with other oxygen sites through the Lennard-Jones interaction. The potential energy between two water molecules, $\alpha$ and $\beta$, is then

$$
E_{\alpha \beta}=4 \epsilon\left[\left(\frac{\sigma}{r_{\mathrm{OO}}}\right)^{12}-\left(\frac{\sigma}{r_{\mathrm{OO}}}\right)^{6}\right]+\sum_{i j} \frac{q_{i} q_{j}}{r_{i j}},
$$

where $r_{\mathrm{OO}}$ is the distance between the two oxygen atoms, the sum is over the charge sites on the two molecules. The potential is characterized by the charges on the sites, $q_{i}$, and the Lennard-Jones parameters $\epsilon$ and $\sigma$ (see Table I). By charge conservation, the charge on the M-site is equal to minus the charge on the hydrogen sites. The geometry of the molecule, including the positions of the M-sites, represents further parameters of the model which are optimized.

The potential is reoptimized to be used with Ewald sums by modifying the Lennard-Jones parameters. All other parameters, including the charge parameter and the geometry, are kept the same, in order to keep the modified version close to the original TIP5P model. The charge parameter is not likely to be a good choice for reoptimization, since, in order to decrease the energy and increase the density as is needed (see the Introduction), the charge would have to be increased. This would lead to a larger dielectric constant ${ }^{1,42}$ and the TIP5P model already has a dielectric constant which is slightly too high. The procedure for modifying the Lennard-Jones parameters can be explained by rewriting the Lennard-Jones interaction, $E_{\mathrm{LJ}}$, as

$$
E_{\mathrm{LJ}}\left(r_{\mathrm{OO}}\right)=4 \epsilon\left[\left(\frac{\sigma}{r_{\mathrm{OO}}}\right)^{12}-\left(\frac{\sigma}{r_{\mathrm{OO}}}\right)^{6}\right]=\frac{A}{r_{\mathrm{OO}}^{12}}-\frac{C}{r_{\mathrm{OO}}^{6}},
$$

where

$$
A=4 \epsilon \sigma^{12}, \quad C=4 \epsilon \sigma^{6},
$$

and equivalently

$$
\sigma=(A / C)^{1 / 6}, \quad \epsilon=C^{2} / 4 A .
$$

The parameters $A$ and $C$, unlike $\epsilon$ and $\sigma$, act as nearly independent parameters. The value of $A$, which characterizes the short-ranged repulsive interactions, is chosen so that the first peak of the $g_{\mathrm{OO}}(r)$ correlation function is in agreement with experiment. The value of $C$, which does not influence the liquid structure strongly, is then adjusted so that the desired values for the energy and pressure (and therefore, the density) are achieved. This is done at $1 \mathrm{~atm}$ and $298 \mathrm{~K}$ and with Ewald sums. This procedure rapidly found values for $A$ and $C$ which optimized the energy, density, and $g_{\text {oo }}(r)$. The new values of $\epsilon$ and $\sigma$ are given in Table I. The new model is named TIP5P-E, to indicate use with Ewald sums. The original TIP5P model has values of $A$ and $C$ equal to 5.45 $\times 10^{5} \AA^{12} \mathrm{kcal} / \mathrm{mol}$ and $590 \AA^{6} \mathrm{kcal} / \mathrm{mol}$, respectively. The TIP5P-E values are $5.54 \times 10^{5} \AA^{12} \mathrm{kcal} / \mathrm{mol}$ and 628 $\AA^{6} \mathrm{kcal} / \mathrm{mol}$, respectively, indicating how small the changes are between TIP5P and TIP5P-E, when viewed in terms of $A$ and $C$. The long-ranged attractive contribution $(C)$ is slightly $(6 \%)$ larger, to lower the potential energy, and the shortranged repulsive contribution is slightly (less than $2 \%$ ) larger to keep the same position in first peak of the $g_{\mathrm{OO}}(r)$ and to give the correct density.

\section{B. Simulation details}

The simulations were done in the isothermal-isobaric (constant T,P,N) ensemble, by coupling to a pressure bath (at 1 atm) and a Nosé-Hoover temperature bath. ${ }^{43-47}$ Simulations were done for systems sizes of 256 and 512 molecules. The Ewald method was implemented using a screening parameter equal to $6 / \mathrm{L}$, where $L$ is the simulation box side length, and a maximum Fourier space vector $\left(K_{\max }\right)$ equal to $2 \pi 5 / L$ and conducting boundary conditions. ${ }^{18}$ The LennardJones interactions were truncated at half the box length and no tail corrections were added. ${ }^{48}$ Each temperature, $T$, was simulated for at least 2 nanoseconds. At the temperatures 0 , 12.5 , and $25.0^{\circ} \mathrm{C}$, the simulations were for 3 nanoseconds. The smaller size system was simulated for longer times (from 3 to 8 nanoseconds) in order to get better estimates of the dielectric constant. The simulations for the dynamical properties (the diffusion constant and the rotational time constant) were done in the $T, V, N$ ensemble at the density given by the $T, P, N$ simulations. The results were averaged from 20 different 10 ps simulations.

Simulations were also carried out using the reaction field method $^{18,23,49}$ in which the long-ranged electrostatic are scaled to go to zero at the cut-off distance, $r_{\text {cut }}$, by

$$
\begin{aligned}
E_{\text {Coulomb }}\left(r_{i j}\right)= & q_{i} q_{j}\left[\frac{1}{r_{i j}}+\frac{\epsilon_{\mathrm{rf}}-1}{2 \epsilon_{\mathrm{rf}}+1} \frac{r_{i j}^{2}}{r_{\mathrm{cut}}^{3}}\right. \\
& \left.-\left(\frac{1}{r_{\mathrm{cut}}}+\frac{\epsilon_{\mathrm{rf}}-1}{2 \epsilon_{\mathrm{rf}}+1} \frac{r_{\mathrm{cut}}^{2}}{r_{\mathrm{cut}}^{3}}\right)\right],
\end{aligned}
$$

where $\epsilon_{\mathrm{rf}}$ is the reaction field dielectric constant, which was set equal to $\infty$. Constant $T, P, N$ simulations using this approach were done at a single temperature and pressure $\left(25^{\circ} \mathrm{C}\right.$ and $1 \mathrm{~atm}$ ) for $1 \mathrm{~ns}$. 
The various thermodynamic and dynamical properties are found from standard formulas. ${ }^{18}$ The heat of vaporization is found from

$$
\Delta H_{\text {vap }}=-\langle E(\text { liquid })\rangle / N+R T,
$$

where $E$ (liquid) is the energy of the liquid with $N$ molecules and $R$ is the ideal gas constant. ${ }^{50}$ The isothermal compressibility is be calculated from

$$
\kappa=-\frac{1}{V}\left(\frac{\partial V}{\partial P}\right)_{N, T}=\frac{1}{k T\langle V\rangle_{N, P, T}}\left(\left\langle V^{2}\right\rangle_{N, P, T}-\langle V\rangle_{N, P, T}^{2},\right.
$$

where $V$ is the volume and $K$ is Boltzmann's constant. ${ }^{18}$ The isothermal compressibility can be found from

$$
\begin{aligned}
\alpha=\frac{1}{V}\left(\frac{\partial V}{\partial T}\right)_{N, P}= & \frac{1}{k T^{2}\langle V\rangle_{N, P, T}}\left(\langle V H\rangle_{N, P, T}\right. \\
& \left.-\langle V\rangle_{N, P, T}\langle H\rangle_{N, P, T}\right),
\end{aligned}
$$

and the heat capacity is found from

$$
C_{P}=\left(\frac{\partial H}{\partial T}\right)_{N, P}=\frac{1}{N k T^{2}}\left(\left\langle H^{2}\right\rangle-\langle H\rangle^{2}\right)+3 R,
$$

where $H$ is $E$ (liquid) $+P V$ and $P$ is pressure. The dielectric constant is found from

$$
\boldsymbol{\epsilon}=\boldsymbol{\epsilon}_{\infty}+\frac{4 \pi}{3 k T\langle V\rangle}\left(\left\langle\boldsymbol{M}^{2}\right\rangle-\langle\boldsymbol{M}\rangle^{2}\right),
$$

where $\boldsymbol{M}$ is the total dipole of the central simulation box and $\epsilon_{\infty}$ is the infinite frequency, or optical, dielectric constant, which for nonpolarizable models is equal to $1 .{ }^{18}$ The diffusion constant is found from the Einstein relation

$$
D=\lim _{t \rightarrow \infty} \frac{1}{6 t}\left\langle\left|\boldsymbol{r}_{i}^{\mathrm{cm}}(t)-\boldsymbol{r}_{i}^{\mathrm{cm}}(0)\right|^{2}\right\rangle,
$$

where $\mathbf{r}_{i}^{\mathrm{cm}}(t)$ is the position of the center-of-mass of molecule $i$ at time $t$. Rotational time constants are found from

$$
C_{l}^{\alpha}(t)=\left\langle P_{l}\left[\boldsymbol{e}_{i}^{\alpha}(t) \cdot \boldsymbol{e}_{i}^{\alpha}(0)\right]\right\rangle,
$$

where $P_{l}$ is a Legendre polynomial and $\boldsymbol{e}_{i}^{\alpha}$ is a unit vector along the principle axis of rotation of molecule $i$. If the $y$ axis is defined as connecting the hydrogen atoms, then the longtime exponential decay of $C_{2}^{Y}(\mathrm{t})$ gives the rotational time constant, $\tau_{\mathrm{NMR}} \cdot{ }^{51}$

\section{RESULTS}

The results labeled TIP5P are for the original TIP5P parameterization using the $9 \AA$ cutoff $^{2}$ and those labeled TIP5P-E are for the new parameterization using Ewald sums and both use 512 molecules, except as indicated. The oxygen-oxygen radial distribution function, $g_{\mathrm{OO}}(r)$, for the TIP5P-E model agrees extremely well with the experimental data from $x$-ray scattering ${ }^{52}$ (Fig. 1). The TIP5P model has been previously demonstrated to give perhaps the most accurate $g_{\mathrm{OO}}(r)$ of any empirical water model. ${ }^{53}$ The new parameterization maintains this level of accuracy in part because this was one of the properties used to fit the new

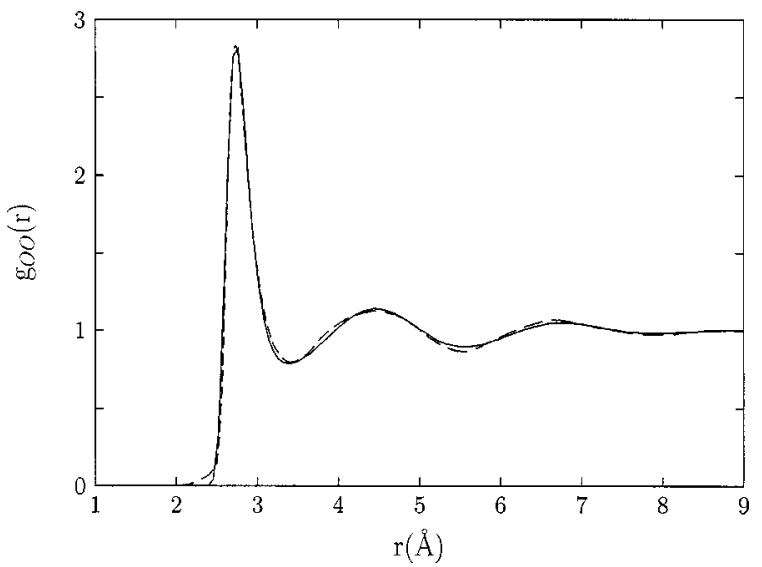

FIG. 1. The oxygen-oxygen radial correlation function, $g_{\mathrm{OO}}(r)$, comparing the TIP5P-E potential simulation results with the $\mathrm{x}$-ray experimental data (Ref. 52).

parameters. The $g_{\mathrm{OO}}$ for the TIP5P and TIP5P-E models are virtually the same (the comparison between the two models is not shown). The $\mathrm{x}$-ray data agrees very well with recent neutron diffraction data ${ }^{54}$ (see Ref. 53).

The dependence of the density with temperature for the TIP5P model is shown in Fig. 2(A), which compares the results using the $9 \AA$ cutoff with 216 and 512 molecules as well as with Ewald sums. Figure 2(B) shows the results of the TIP5P-E model (using Ewald) for 512 molecules, compared to the TIP5P model. The densities for the TIP5P and
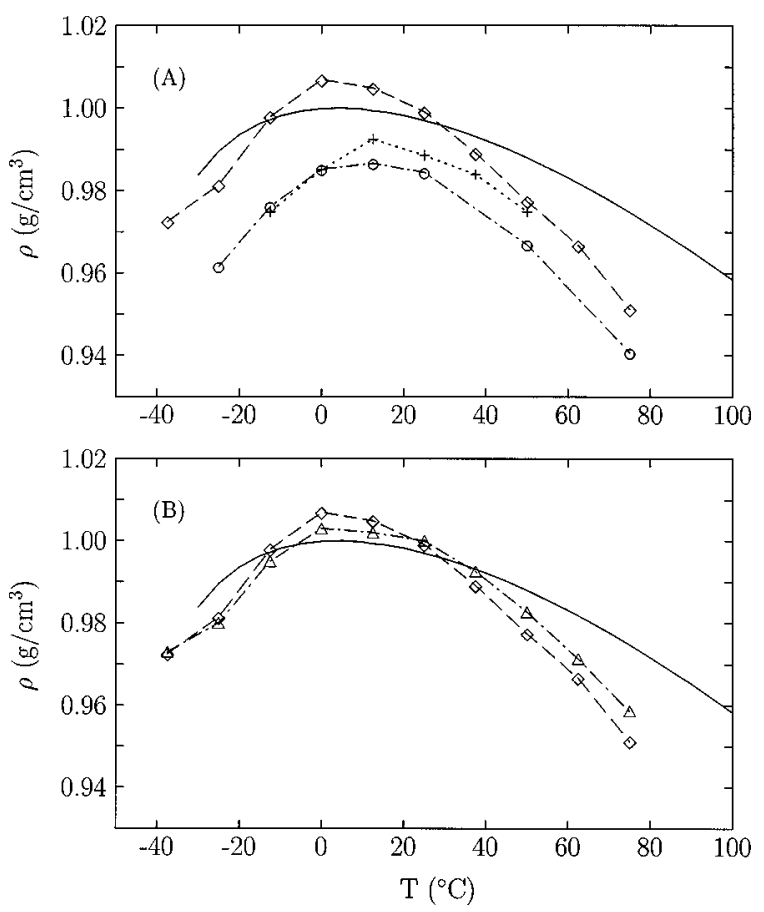

FIG. 2. Density as a function of temperature for (A) the TIP5P model with 512 molecules using a 9 A cut-off (diamonds) (Ref. 2), 216 molecules using a $9 \AA$ cutoff (crosses) (Ref. 2), and 512 molecules using Ewald (circles) (Ref. 38) and (B) the TIP5P model with 512 molecules using a $9 \AA$ cutoff (diamonds) (Ref. 2), the TIP5P-E model with 512 molecules using Ewald (circles), and the TIP5P-E model with 256 molecules using Ewald (crosses). In both (A) and (B) the solid line without symbols is the experimental data. (Ref. 55). 
TABLE II. Properties of the TIP5P-E model at various temperatures at a pressure of $1 \mathrm{~atm}$, showing the results with two different system sizes (256 and 512 molecules) as well as the TIP5P results (with 512 molecules) from Ref. 2 and the experimental data.

\begin{tabular}{|c|c|c|c|c|c|c|c|}
\hline $\begin{array}{c}T \\
\left({ }^{\circ} \mathrm{C}\right)\end{array}$ & & $\begin{array}{c}\rho \\
\left(\mathrm{g} / \mathrm{cm}^{3}\right)\end{array}$ & $\begin{array}{c}\Delta H_{\mathrm{vap}} \\
(\mathrm{kcal} / \mathrm{mol})\end{array}$ & $\begin{array}{c}C_{P} \\
(\mathrm{cal} / \mathrm{mol} / \mathrm{deg})\end{array}$ & $\begin{array}{l}10^{5} \alpha \\
\left({ }^{\circ-1}\right)\end{array}$ & $\begin{array}{c}10^{6} \kappa \\
\left(\mathrm{atm}^{-1}\right)\end{array}$ & $\epsilon$ \\
\hline \multirow[t]{2}{*}{-37.5} & TIP5P-E 512 & $0.973(2)$ & 12.03(3) & 19(2) & $12(21)$ & 18(3) & \\
\hline & TIP5P 512 & $0.9725(3)$ & $12.084(3)$ & & & & \\
\hline \multirow[t]{3}{*}{-25.0} & TIP5P-E 512 & $0.980(3)$ & $11.67(3)$ & $22(1)$ & $11(14)$ & 29(3) & \\
\hline & TIP5P & $0.9814(4)$ & $11.823(7)$ & $43.0(2)$ & $-125(1)$ & $17(1)$ & \\
\hline & experiment & $0.9896^{\mathrm{a}}$ & & & $-95.59^{a}$ & $71.88^{\mathrm{a}}$ & \\
\hline \multirow[t]{4}{*}{-12.5} & TIP5P-E 512 & $0.995(2)$ & $11.27(2)$ & $32(3)$ & $-50(30)$ & $48(4)$ & \\
\hline & TIP5P-E 256 & $0.994(3)$ & $11.26(3)$ & $42(4)$ & $-107(48)$ & $55(9)$ & \\
\hline & TIP5P & $0.9979(8)$ & $11.367(8)$ & $39.4(3)$ & $-105(3)$ & 24(1) & \\
\hline & experiment & $0.9973^{\mathrm{a}}$ & & & $-36.62^{\mathrm{a}}$ & $58.27^{\mathrm{a}}$ & \\
\hline \multirow[t]{4}{*}{0.0} & TIP5P-E 512 & $1.003(1)$ & $10.924(7)$ & $31(2)$ & $-18(22)$ & $52(4)$ & $95(14)$ \\
\hline & TIP5P-E 256 & $1.0004(8)$ & $10.911(6)$ & $33(1)$ & $-31(9)$ & $57(3)$ & $99(8)$ \\
\hline & TIP5P 512 & $1.007(1)$ & $11.041(8)$ & $33.8(5)$ & $-32(5)$ & $31(1)$ & $92(2)$ \\
\hline & experiment & $0.9998^{\mathrm{a}}$ & $10.76^{\mathrm{b}}$ & $18.16^{\mathrm{c}}$ & $-6.80^{\mathrm{a}}$ & $51.56^{\mathrm{a}}$ & $87.74^{\mathrm{d}}$ \\
\hline \multirow[t]{4}{*}{12.5} & TIP5P-E 512 & $1.0039(6)$ & $10.633(4)$ & $29(1)$ & $18(9)$ & $53(3)$ & $90(9)$ \\
\hline & TIP5P-E 256 & $1.002(2)$ & $10.623(6)$ & $30.1(4)$ & 21(4) & $57(2)$ & $92(7)$ \\
\hline & TIP5P 512 & $1.005(1)$ & $10.735(7)$ & $30.9(8)$ & $33(7)$ & $36(1)$ & \\
\hline & experiment & $0.9994^{\mathrm{a}}$ & $10.64^{\mathrm{b}}$ & $18.04^{c}$ & $12.04^{\mathrm{a}}$ & $47.86^{\mathrm{a}}$ & $83.02^{\mathrm{d}}$ \\
\hline \multirow[t]{4}{*}{25.0} & TIP5P-E 512 & $1.0000(5)$ & $10.377(4)$ & $27.2(6)$ & $49(6)$ & $52(3)$ & $92(14)$ \\
\hline & TIP5P-E 256 & $0.998(1)$ & $10.359(2)$ & 28.3(5) & $60(4)$ & 61(4) & $86(6)$ \\
\hline & TIP5P 512 & $0.999(1)$ & $10.46(1)$ & $29.1(8)$ & $63(6)$ & $41(2)$ & $82(2)$ \\
\hline & experiment & $0.9970^{\mathrm{a}}$ & $10.51^{\mathrm{b}}$ & $18.00^{c}$ & $25.7^{\mathrm{a}}$ & $45.85^{\mathrm{a}}$ & $78.3^{\mathrm{d}}$ \\
\hline \multirow[t]{4}{*}{37.5} & TIP5P-E 512 & $0.9926(6)$ & $10.133(3)$ & $26.6(5)$ & $69(10)$ & $58(3)$ & $80(2)$ \\
\hline & TIP5P-E 256 & $0.991(1)$ & $10.116(3)$ & 27.4(4) & $87(9)$ & $66(3)$ & $85(3)$ \\
\hline & TIP5P & $0.989(1)$ & $10.207(6)$ & 27.6(3) & $87(5)$ & $47(1)$ & \\
\hline & experiment & $0.9931^{\mathrm{a}}$ & $10.38^{\mathrm{b}}$ & $17.99^{c}$ & $36.58^{\mathrm{a}}$ & $44.91^{\mathrm{a}}$ & $74.11^{\mathrm{d}}$ \\
\hline \multirow[t]{4}{*}{50.0} & TIP5P-E 512 & $0.9827(6)$ & $9.910(3)$ & $25.5(9)$ & $91(13)$ & $60(3)$ & $77(9)$ \\
\hline & TIP5P-E 256 & $0.9797(7)$ & $9.889(6)$ & $26.9(1)$ & $120(13)$ & 75(4) & $83(3)$ \\
\hline & TIP5P 512 & $0.978(2)$ & $9.967(6)$ & $27(1)$ & $92(11)$ & $56(4)$ & $75(2)$ \\
\hline & experiment & $0.9880^{\mathrm{a}}$ & $10.25^{\mathrm{b}}$ & $18.00^{\mathrm{c}}$ & $45.76^{\mathrm{a}}$ & $44.76^{\mathrm{a}}$ & $69.91^{\mathrm{d}}$ \\
\hline \multirow[t]{3}{*}{62.5} & TIP5P-E 512 & $0.9714(6)$ & $9.697(3)$ & $24.9(5)$ & $106(9)$ & $64(4)$ & $80(2)$ \\
\hline & TIP5P 512 & $0.967(2)$ & $9.744(6)$ & $25.9(9)$ & $110(10)$ & $59(3)$ & \\
\hline & experiment & $0.9819^{\mathrm{a}}$ & $10.12^{\mathrm{b}}$ & $18.02^{\mathrm{c}}$ & $53.86^{\mathrm{a}}$ & $45.22^{\mathrm{a}}$ & $66.17^{\mathrm{d}}$ \\
\hline \multirow[t]{3}{*}{75.0} & TIP5P-E 512 & $0.9586(7)$ & $9.493(5)$ & $24(1)$ & $118(14)$ & $67(4)$ & $72(1)$ \\
\hline & TIP5P 512 & $0.9512(9)$ & $9.519(7)$ & $25.9(8)$ & $127(7)$ & $65(3)$ & $69(2)$ \\
\hline & experiment & $0.9748^{\mathrm{a}}$ & $9.991^{b}$ & $18.05^{\mathrm{c}}$ & $61.27^{\mathrm{a}}$ & $46.22^{\mathrm{a}}$ & $62.43^{\mathrm{d}}$ \\
\hline
\end{tabular}

${ }^{\mathrm{a}}$ Reference 55

${ }^{\mathrm{b}}$ Reference 57.

${ }^{\mathrm{c}}$ Reference 56

${ }^{\mathrm{d}}$ Reference 58 .

the TIP5P-E models are almost identical and the density maximum for both is near the same temperature. The coefficient of thermal expansion, $\alpha$, changes from being negative at $0{ }^{\circ} \mathrm{C}$ to positive at $12.5^{\circ} \mathrm{C}$, indicating that the temperature of maximum density (TMD) is between these two temperatures. There are some differences at higher temperatures, with the TIP5P-E model in closer agreement with experiment. The average error in the density over the temperature range of -37.5 to $62.5^{\circ} \mathrm{C}$ is $0.006 \mathrm{~g} / \mathrm{cm}^{3}$ for the TIP5P model, ${ }^{2}$ while for the TIP5P-E model it is $0.004 \mathrm{~g} / \mathrm{cm}^{3}$. Other thermodynamic properties over a range of temperature for the TIP5P-E model are shown on Table II. A comparison is made with the TIP5P model results ${ }^{2,4}$ as well as experimental data $^{55-60}$ (which for some temperatures are interpolations between reported data points). Results using two different system sizes are reported ( 256 and 512 molecules) at some temperatures. The dependence on system size when used with Ewald is not that significant, in agreement with Ref. 38, but the two results do provide independent estimates of the re- ported properties. This is useful especially for the dielectric constant, which requires long simulation times to get adequately sampled values.

The TIP5P results are for a system size of 512 molecules, except for the dielectric constant which used 216 molecules with an $8 \AA$ cutoff (and the density set the experimental density, not the density given by the model at $1 \mathrm{~atm})^{2}$ and the diffusion constant which used 267 molecules and a 9 $\AA$ cutoff (and a density as given by the model with 512 molecules). ${ }^{2}$ Comparisons between the TIP5P-E and TIP5P model are further complicated by small differences between the methods used to calculate the various properties. The heat capacity $\left(C_{p}\right)$, isothermal compressibility $(\kappa)$, and coefficient of thermal expansion $(\alpha)$ are all calculated here using fluctuation formulas. ${ }^{18}$ In Ref. $2, C_{p}$, and $\alpha$ were computed using finite difference approximations to the temperature derivatives. The dielectric constant was calculated by applying a small electric field and calculating the response of the total dipole moment $(\mathbf{M})$ of the system in 


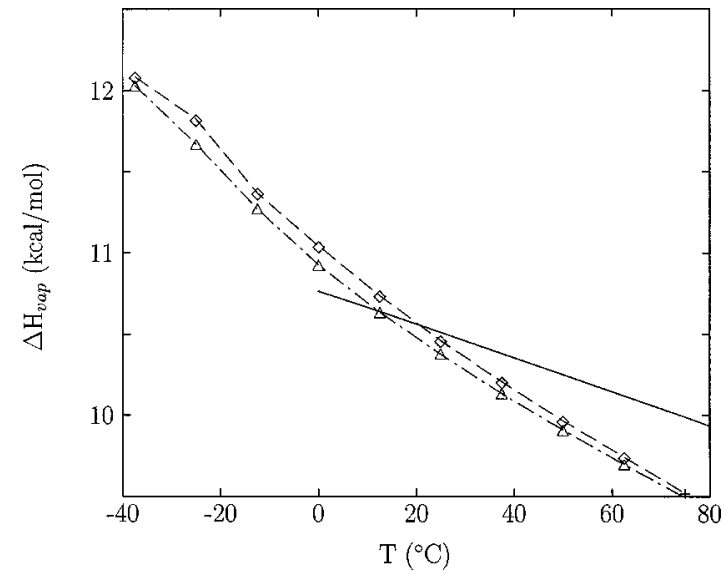

FIG. 3. Heat of vaporization for the TIP5P-E model (triangles and dotdashed line), the TIP5P model (diamonds and dashed line) (Ref. 2) and the experimental value (Ref. 57).

Ref. 2 (and at the experimental rather than calculated density). Our results calculate the dielectric through fluctuations in $\mathbf{M}$ (and at the model's density at the given temperature.)

The enthalpy of vaporization for the TIP5P-E model is slightly higher than the TIP5P result, which means the average potential energy of the liquid, $E$ (liquid), is underestimated (Table II and Fig. 3). At $25^{\circ}$, E(liquid) is $-9.78 \mathrm{kcal} /$ mol for the TIP5P-E model and $-9.87 \mathrm{kcal} / \mathrm{mol}$ for the TIP5P model. The TIP5P-E value for $E$ (liquid), and therefore, $\Delta H_{\mathrm{vap}}$, is always less in magnitude than that of TIP5P. The TIP5P value agrees better with the experimental value at $25^{\circ}$ and at higher temperatures. At lower temperatures, the TIP5P-E results are closer to experiment. The $H_{\text {vap }}$ for the TIP5P and TIP5P-E as a function of temperature are almost parallel, which means that they have the about same heat capacity, $C_{P}$. Both have a heat capacity which is too large compared to experiment. Other models, including TIP3P and TIP4P, ${ }^{39,61}$ have smaller heat capacities in closer agreement with experiment, but have a much less accurate temperature dependence of the density. Other models which have a near correct temperature of maximum density, TMD, are the
TIP4P-FQ and POL5/TZ models and these models also overestimate the heat capacity. ${ }^{7,62}$ For the TIP5P, TIP4P-FQ, and POL5/TZ models, the enthalpy then changes too strongly with temperature and this may be necessary to have a good density dependence (and an accurate TMD). These models are fixed geometry models and therefore do not have contributions to the heat capacity coming from the bond angle and bond stretch degrees-of-freedom. For the thermodynamic properties, the isothermal compressibility, $\kappa$, and the coefficient of thermal expansion, $\alpha$, the TIP5P-E and TIP5P models give very similar results.

The results found using the reaction field method [Eq. (5)] are in close agreement with the Ewald results. The density is $0.998 \pm 0.001 \mathrm{~g} / \mathrm{cm}^{3}$ and the $\Delta H_{\text {vap }}$ is $10.354 \pm 0.003$ $\mathrm{kcal} / \mathrm{mol}$. The close agreement between the Ewald and reaction field methods for TIP5P-E has also been demonstrated for TIP5P $\mathrm{P}^{38}$ and other water models. ${ }^{23}$

Values for the dynamical properties of the diffusion constant, $D$, and the nuclear magnetic resonance (NMR) relaxation time, $\tau_{\mathrm{NMR}}$, are given in Table III. The diffusion constants for the TIP5P-E is slightly higher than the results for the TIP5P over the range of temperatures examined. Some of the differences between the TIP5P and TIP5P-E results are due to differences in the implementation of the constant temperature dynamics. The TIP5P values were calculated using Berendsen method, ${ }^{63}$ while the present results used NoséHoover thermostating. ${ }^{46,47}$ The Nosé-Hoover method is a gentler method for modifying the velocities and is a preferred method for performing constant $T, P, N$ simulations for use in calculating dynamical properties. ${ }^{48}$ As a comparison, constant $E, V, N$ simulations at $25^{\circ} \mathrm{C}$ gave the same results as those presented on Table III. We also calculated the diffusion constant for TIP5P at $-37.5,25$, and $75^{\circ} \mathrm{C}$ using Nosé-Hoover thermostating. The value at $25^{\circ} \mathrm{C}$ is $2.8 \pm 0.2$ $\times 10^{-9} \mathrm{~m}^{2} / \mathrm{s}$, within error bars of both the previous value for TIP5P and the value for TIP5P-E. At the higher temperature of $75^{\circ} \mathrm{C}$, the Nosé-Hoover method gives a value of 7.6 $\pm 0.2 \times 10^{-9} \mathrm{~m}^{2} / \mathrm{s}$, slightly higher than the value reported by Mahoney and Jorgensen. The diffusion constants are rela-

TABLE III. Dynamical properties for TIP5P-E and TIP5P models and the experimental values at $1 \mathrm{~atm}$.

\begin{tabular}{|c|c|c|c|c|c|c|}
\hline \multirow{2}{*}{$\begin{array}{l}T \\
\left({ }^{\circ} \mathrm{C}\right)\end{array}$} & \multicolumn{3}{|c|}{$\begin{array}{c}D \\
\left(10^{-9} / \mathrm{m}^{2} / \mathrm{s}\right)\end{array}$} & \multicolumn{3}{|c|}{$\begin{array}{c}\tau_{\mathrm{NMR}} \\
(\mathrm{ps})\end{array}$} \\
\hline & TIP5P-E & TIP5P & Expt. & TIP5P-E & TIP5P & Expt. \\
\hline-37.5 & $0.09(2)$ & $0.070(8)$ & & $63(10)$ & $69(7)$ & \\
\hline-25.0 & $0.17(2)$ & $0.14(4)^{\mathrm{a}}$ & & $28(2)$ & & \\
\hline-12.5 & $0.48(5)$ & $0.43(6)^{\mathrm{a}}$ & $0.66^{\mathrm{b}}$ & $10.3(9)$ & & \\
\hline 0.0 & $1.2(1)$ & $1.06(8)^{\mathrm{a}}$ & $1.10^{\mathrm{c}}$ & $4.1(3)$ & & \\
\hline 12.5 & $1.9(1)$ & $1.9(2)^{\mathrm{a}}$ & $1.64^{\mathrm{c}}$ & $2.3(1)$ & & $3.44^{\mathrm{d}}$ \\
\hline 25.0 & $2.8(1)$ & $2.62(8)^{\mathrm{a}}$ & $2.30^{\mathrm{c}}$ & $1.55(4)$ & $1.58(5)$ & $2.46^{\mathrm{d}}$ \\
\hline 37.5 & $3.88(6)$ & $3.7(1)^{\mathrm{a}}$ & $3.07^{\mathrm{c}}$ & $1.03(3)$ & & $1.92^{\mathrm{d}}$ \\
\hline 50.0 & $5.2(2)$ & $4.7(1)^{\mathrm{a}}$ & $3.95^{\mathrm{c}}$ & $0.74(1)$ & & $1.66^{\mathrm{d}}$ \\
\hline 62.5 & $6.4(2)$ & $6.3(1)^{\mathrm{a}}$ & $4.96^{\mathrm{c}}$ & $0.58(1)$ & & $1.41^{\mathrm{d}}$ \\
\hline 75.0 & $8.0(2)$ & $6.8(2)^{\mathrm{a}}$ & $6.08^{\mathrm{c}}$ & $0.44(2)$ & $0.47(2)$ & $1.12^{\mathrm{d}}$ \\
\hline
\end{tabular}

${ }^{\mathrm{a}}$ Reference 4 .

${ }^{\mathrm{b}}$ Reference 59.

${ }^{c}$ Reference 60

${ }^{\mathrm{d}}$ Reference 66 
TABLE IV. Thermodynamic properties for the TIP5P-E model as a function of pressure at a temperature of $25^{\circ} \mathrm{C}$.

\begin{tabular}{rlllll}
\hline \hline $\begin{array}{c}P \\
(\mathrm{~atm})\end{array}$ & $\begin{array}{c}\rho \\
\left(\mathrm{g} / \mathrm{cm}^{3}\right)\end{array}$ & $\begin{array}{c}\Delta H_{\mathrm{vap}} \\
(\mathrm{kcal} / \mathrm{mol})\end{array}$ & $\begin{array}{c}C_{P} \\
(\mathrm{cal} / \mathrm{mol} / \mathrm{deg})\end{array}$ & $\begin{array}{c}10^{5} \alpha \\
\left(\mathrm{deg}^{-1}\right)\end{array}$ & $\begin{array}{c}10^{6} \kappa \\
\left(\mathrm{atm}^{-1}\right)\end{array}$ \\
\hline 1 & $1.0000(5)$ & $10.377(4)$ & $27.2(6)$ & $49(6)$ & $52(3)$ \\
1000 & $1.0490(9)$ & $10.425(5)$ & $26(1)$ & $49(12)$ & $39(3)$ \\
2000 & $1.0894(5)$ & $10.45(1)$ & $25(1)$ & $43(9)$ & $32(2)$ \\
3000 & $1.123(1)$ & $10.481(6)$ & $23.2(8)$ & $55(2)$ & $27.4(8)$ \\
4000 & $1.1530(9)$ & $10.502(7)$ & $23(1)$ & $53(10)$ & $24.3(4)$ \\
5000 & $1.1802(4)$ & $10.523(4)$ & $23.0(6)$ & $59(10)$ & $22(2)$ \\
6000 & $1.204(1)$ & $10.543(5)$ & $22.8(7)$ & $55(4)$ & $19(1)$ \\
8000 & $1.245(1)$ & $10.575(3)$ & $23.0(4)$ & $59(7)$ & $15(1)$ \\
10000 & $1.2810(9)$ & $10.600(2)$ & $23(1)$ & $67(8)$ & $14.1(5)$ \\
\hline \hline
\end{tabular}

tively close to the experimental values and are closer than the values for SPC, TIP3P, and TIP4P., ${ }^{4,64,65}$ The quantity $\tau_{\mathrm{NMR}}$ gives the time scale for rotations about the axis connecting the hydrogen atoms (the $y$ axis) and can be measured using nuclear magnetic resonance (NMR). The TIP5P and TIP5P-E results for the quantity are again close for the three temperatures examined for TIP5P. The TIP5P-E results may be slightly smaller, indicating faster rotational dynamics. Our result for TIP5P at $25^{\circ}$ agrees fairly well with the result of Stern et al., who report $1.4 \pm 0.1 \mathrm{ps}^{3}{ }^{3}$ That study used Ewald, which may be the reason for any small difference with our result of $1.58 \pm 0.5 \mathrm{ps}$. The TIP5P and TIP5P-E values are smaller than the experimental results, ${ }^{66}$ indicating, along with the diffusion constant, faster dynamics. However, the value is closer to experiment than many models. Among the commonly used nonpolarizable models, only the SPC/E model gives values of $\mathrm{D}$ and $\tau_{\mathrm{NMR}}$ closer to experiment ( $D$ $=2.4 \pm 0.4 \times 10^{-9} \mathrm{~m}^{2} / \mathrm{s}$ and $\tau_{\mathrm{NMR}}=1.9 \pm 0.1$ at $\left.25^{\circ} \mathrm{C}\right) .{ }^{67}$

The thermodynamic properties as a function of pressure at $25^{\circ} \mathrm{C}$ are given in Table IV. The density as a function of pressure is shown in Fig. 4, which also shows the results for TIP5P ${ }^{2}$ and the experimental results. ${ }^{68}$ The two models give very close results and the agreement with experiment is good.

The dielectric constants for the TIP5P and TIP5P-E models are equivalent within the bars and close to the ex-

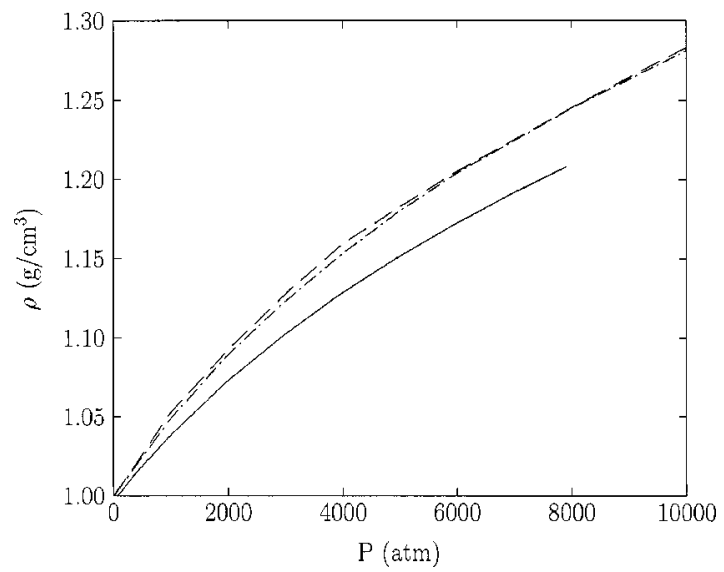

FIG. 4. Density as a function of pressure for the TIP5P-E model (dotdashed line), TIP5P model (dashed line) (Ref. 2), and the experimental value (Ref. 68). perimental values, as been reported previously. ${ }^{2}$ The agreement with experiment is surprising given the dipole moment of the models, which is only 2.29 Debye (D). An analysis of 6 water models by Sprik demonstrated a correlation between the dipole moment, $\mu$, and the dielectric constant, $\epsilon$, of the models. ${ }^{42}$ Models with a dipole moment around 2.5-2.6 Debye tend to have a dielectric constant around 80 . This analysis resulted in $2.6 \mathrm{D}$ being a target value for water potentials. A similar correlation between $\mu$ and $\epsilon$ is seen in subsequent analyses of 14 water models by Wallqvist and Mountain ${ }^{1}$ and 16 models by Soetens et al. ${ }^{69}$ The TIP5P value for $\epsilon$, in light of the model's relatively small dipole moment, is one of the largest deviations from the $\mu \epsilon$ correlation. Another apparent anomaly is TIP3P, which has $\mu=2.35 \mathrm{D}$ and $\epsilon=95 .{ }^{70}$ The TIP3P and TIP5P results are more recent than the analyses presented in Refs. 1, 42, and 69 and are, therefore, not presented in those plots.

The large dielectric constant of TIP3P and TIP5P can be explained from the results of Carnie and Patey, ${ }^{41}$ which examined a waterlike model of hard spheres with embedded dipoles and quadrupoles. This study showed that the quadrupole interactions strongly quench the dipolar correlations and, as a consequence, the dielectric constant of the liquid decreases considerably as the quadrupole interactions are increased. For the various water potentials, the effects of the quadrupole interactions on $\epsilon$ can be examined by comparing models which have similar dipole moments but different quadrupoles (Table V). Water models can have similar values of $\mu$ but different quadrupoles by having a different charge site geometry or by having polarizable point dipoles. There are several models with dipole moments from 2.2 to 2.3 Debye and several with dipole moments, by design, around 2.6 Debye. For the polarizable models, this dipole moment is an average, including the induced dipole moment, at $25^{\circ} \mathrm{C}$ and $1 \mathrm{~atm}$. The quadrupoles are relative to the center of mass and the $z$ axis is in the dipole moment direction, the $y$ axis connects the two hydrogen atoms, and $x$ axis is out of the plane of the molecule. For the fluctuating charge model (TIP4P-FQ), the quadrupole moments also include an induced part. For a water molecule, the quadrupole tensor can be approximated by ${ }^{41}$ 
TABLE V. Dipole and quadrupole moments and the dielectric constant for liquid water at $25^{\circ} \mathrm{C}$ and 1 atm.

\begin{tabular}{|c|c|c|c|c|c|c|}
\hline & $\begin{array}{c}\mu \\
\text { (D) }\end{array}$ & $\begin{array}{c}\mathrm{Q}_{x x} \\
(\mathrm{D} \AA \AA)\end{array}$ & $\begin{array}{c}\mathrm{Q}_{y y} \\
(\mathrm{D} \stackrel{\AA}{\mathrm{A}})\end{array}$ & $\begin{array}{c}\mathrm{Q}_{z z} \\
(\mathrm{D} A)\end{array}$ & $\begin{array}{c}\mathrm{Q}_{T} \\
(\mathrm{D} \AA)\end{array}$ & $\epsilon$ \\
\hline TIP4P $\mathrm{P}^{\mathrm{a}}$ & 2.177 & -2.089 & 2.204 & -0.114 & 2.147 & $53(2)^{b}$ \\
\hline $\mathrm{MCY}^{\mathrm{c}}$ & 2.193 & -2.765 & 3.154 & -0.389 & 2.960 & $34(1)^{\mathrm{d}}$ \\
\hline $\mathrm{SPC}^{\mathrm{e}}$ & 2.274 & -1.823 & 2.115 & -0.292 & 1.969 & $61(1)^{\mathrm{f}}$ \\
\hline TIP5P & 2.29 & -1.48 & 1.65 & -0.170 & 1.57 & $82(2)^{g}$ \\
\hline TIP3P $\mathrm{P}^{\mathrm{h}}$ & 2.35 & -1.68 & 1.76 & -0.088 & 1.72 & $95^{\mathrm{i}}$ \\
\hline $\mathrm{SPC} / \mathrm{E}^{\mathrm{j}}$ & 2.351 & -1.885 & 2.186 & -0.302 & 2.036 & $71(1)^{\mathrm{k}}$ \\
\hline $\mathrm{WK}^{1}$ & 2.596 & -2.493 & 2.626 & -0.134 & 2.560 & $80(8)^{1}$ \\
\hline $\mathrm{COS} / \mathrm{B} 2^{\mathrm{m}}$ & 2.62 & -1.658 & 1.925 & -0.267 & 1.792 & $122^{\mathrm{m}}$ \\
\hline $\mathrm{RPOL}^{\mathrm{n}}$ & 2.62 & -1.622 & 1.884 & -0.262 & 1.753 & $106(18)^{\circ}$ \\
\hline SRWK-Pol ${ }^{p}$ & 2.63 & -2.323 & 2.630 & -0.307 & 2.477 & $86(10)^{\mathrm{p}}$ \\
\hline TIP4P-FQ ${ }^{q}$ & $2.641^{\mathrm{q}}$ & $-2.51^{\mathrm{r}}$ & $2.64^{\mathrm{r}}$ & $-0.13^{\mathrm{r}}$ & 2.58 & $79(8)^{\mathrm{q}}$ \\
\hline Experiment, gas phase & $1.855^{\mathrm{s}}$ & $-2.50^{\mathrm{t}}$ & $2.63^{\mathrm{t}}$ & $-0.13^{\mathrm{t}}$ & 2.57 & \\
\hline$a b$ initio, liquid ${ }^{\mathrm{u}}$ & 2.95 & -3.16 & 3.38 & -0.22 & 3.27 & \\
\hline$a b$ initio, liquid $^{\mathrm{v}}$ & 2.43 & -2.67 & 2.77 & -0.10 & 2.72 & \\
\hline Experiment, liquid & & & & & & $78.36^{\mathrm{w}}$ \\
\hline
\end{tabular}

${ }^{\mathrm{a}}$ Reference 61 .

${ }^{\mathrm{b}}$ Reference 80 .

${ }^{\mathrm{c}}$ Reference 81 .

${ }^{\mathrm{d}}$ Reference 82 .

${ }^{\mathrm{e}}$ Reference 83.

${ }^{\mathrm{f}}$ Reference 84.

${ }^{g}$ Reference 2.

heference 39.

${ }^{\mathrm{i}}$ Reference 70.

${ }^{\mathrm{j}}$ Reference 64.

${ }^{\mathrm{k}}$ Reference 87.

${ }^{\mathrm{l} R e f e r e n c e} 65$.

$$
\boldsymbol{Q}=\left(\begin{array}{ccc}
-Q_{T} & 0 & 0 \\
0 & Q_{T} & 0 \\
0 & 0 & 0
\end{array}\right)
$$

This approximation is reasonable for the water models listed on Table V, in which $Q_{x x} \approx-Q_{y y}$ and $Q_{z z} \approx 0$. The quadrupoles for each model can, therefore, be approximated using the single quantity

$$
Q_{T}=\frac{1}{2}\left(\left|Q_{x x}\right|+\left|Q_{y y}\right|\right)=\frac{1}{2}\left(-Q_{x x}+Q_{y y}\right) .
$$

The value of $\epsilon$ for the various water models versus the strength of the quadrupole is shown on Fig. 5. Two sets of values are shown, for six models with $\mu$ from 2.2 to $2.3 \mathrm{D}$ (the average of $\mu$ among these models is $2.273 \mathrm{D}$ ) and for five with $\mu$ near $2.6 \mathrm{D}$ (the average among these models is $2.621 \mathrm{D})$. Straight line fits through the two sets are also shown. It is apparent that, like the Carnie-Patey study, $\epsilon$ decreases as the quadrupole increases. There appears to be a similar dependence on $\epsilon$ with $Q_{T}$ for the two data sets with different dipoles. This dependence explains why the TIP5P model (and the TIP5P-E model) has a dielectric constant about 80 , despite having a relatively small dipole moment, since it also has a small $Q_{T}$. This analysis includes both polarizable models (COS/B2, ${ }^{71}$ RPOL, ${ }^{72}$ SRWK-Pol, ${ }^{42}$ TIP4P-FQ ${ }^{73}$ ) and nonpolarizable models. The value of $\epsilon$ correlates with the $Q_{x x}$ and $Q_{y y}$ as well as $Q_{T}$, but does not correlate well with $Q_{z z}$, in part because $Q_{z z}$ is an order a magnitude smaller and also since the size of $Q_{z z}$ does not correlate with the size of $Q_{x x}$ or $Q_{y y}$.

\author{
${ }^{\mathrm{m}}$ Reference 71 \\ ${ }^{\mathrm{n}}$ Reference 72. \\ ${ }^{\circ}$ Reference 67. \\ ${ }^{\mathrm{P}}$ Reference 42. \\ ${ }^{\mathrm{q}}$ Reference 73. \\ ${ }^{\mathrm{r}}$ Reference 7. \\ ${ }^{\mathrm{s}}$ Reference 85. \\ ${ }^{\mathrm{t}}$ Reference 86. \\ "Reference 77. \\ ${ }^{v}$ Reference 76. \\ ${ }^{\mathrm{w}}$ Reference 58 .
}

The size of the dielectric response is a combination of the size of the dipole moment and the size of the fluctuations, as is indicated by Eq. (10). A water model can have a large dielectric response by having a large dipole moment or by having a smaller dipole moment which undergoes large fluctuations. The models on the left side of Fig. 5 with $\epsilon$ above 80 and $Q_{T}=1.6$ to $1.7 \mathrm{D} \AA$ (TIP3P and TIP5P) can be termed small dipole, large fluctuation models. The models on the right side with $\epsilon$ around 80 and $Q_{T}$ around $2.5 \mathrm{Q} \AA$ (WK, SWRK-Pol, TIP4P-FQ) are then large dipole, small fluctuation models. (As far as we are aware, there are no models

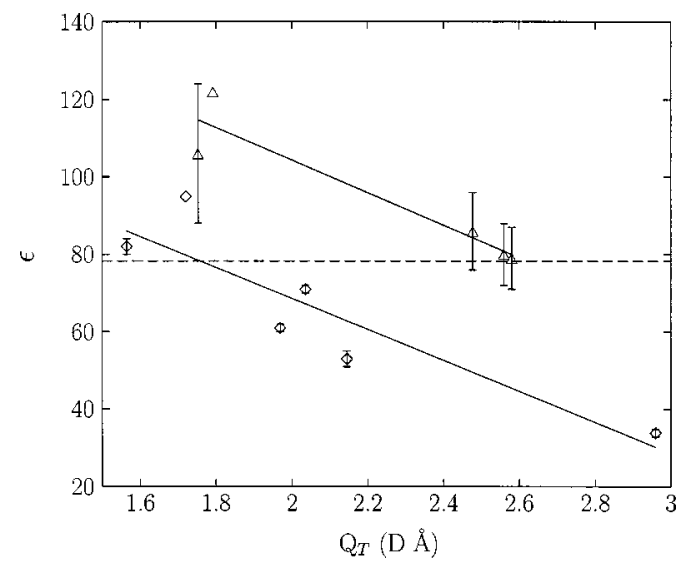

FIG. 5. Dielectric constant as a function of quadrupole moment, $Q_{T}$, for water models with a dipole moment near $2.2 \mathrm{D}$ (diamonds) and with a dipole moment near $2.6 \mathrm{D}$ (triangles). The dash line is at $\epsilon=78.3$. 
with a dipole smaller than $2.29 \mathrm{D}$ or larger than $2.64 \mathrm{D}$ that have $\epsilon$ near $80 .{ }^{1,69}$ ) It is difficult to assess which limit is more physical, since the dipole moment, or the quadrupoles, of a water molecule in the liquid cannot be unambiguously assigned. $^{7,74-77}$ This can be seen in Table V, which also shows the results for $\mu$ and $Q$ from $a b$ initio calculations. ${ }^{76,77}$ The two studies give very different moments depending on the way the electronic density is partitioned to individual molecules. However, it does appear that magnitudes of the quadrupole moments are larger in the liquid than in the gas phase. The TIP3P and TIP5P models have quadrupole moments which are lower than the gas-phase values.

For the type of potentials listed on Table V, the analysis presented on Fig. 5 suggests a way to choose optimal values for $\mu$ and $Q_{T}$ for use in the design of water potentials. In optimizing a potential to get a good dielectric constant, one has to pick good values for the pair of $\mu$ and $Q_{T}$ and not just $\mu$ alone. The two straight line fits shown in Fig. 5 have almost identical slopes. This suggests that the dielectric constant data can be fit to a simple form. A fit for all eleven $\mu$, $Q_{T}$, and $\epsilon$ values listed on Table $\mathrm{V}$ gives

$$
\epsilon\left(\mu, Q_{T}\right)=-85.0+98.0 \mathrm{D}^{-1} \mu-35.7 \quad(\mathrm{D} \AA)^{-1} Q_{T} .
$$

There is no reason to expect the dependence of $\epsilon$ on $\mu$ and $Q_{T}$ to be linear (and certainly the $\mu$ goes to zero limit is unphysical), but over the range of values typical for water models it is appears to be a good approximation. Equation (15) could be used in the development of water models to provide a quick estimate of the dielectric constant. Notice that the $\mu$ dependence is about three times larger than the $Q_{T}$ dependence, but the quadrupole does still influence the dielectric constant. For most of these models, the only interactions on the hydrogen atoms are electrostatic, so all nonisotopic terms in the potential are electrostatic. Therefore, the largest nonisotropic potential energy term, after the dipole, is the quadrupole and it is reasonable that the quadrupole has such a large effect on $\epsilon$. Other short-ranged repulsive and attractive forces, as commonly treated with Lennard-Jones interactions, are usually taken to be only on the oxygen sites. These interactions, if included on more than one site, or other nonisotropic interactions would influence the dielectric response. ${ }^{78}$ If these nonisotropic interactions are large, they would change the optimal values for $\mu$ and $Q_{T}$.

\section{CONCLUSION}

The modified version of the TIP5P model, constructed by reoptimizing the two Lennard-Jones parameters, appears to work as well, when used with Ewald sums, as the original TIP5P model, when used with a $9 \AA$ cutoff. This model was constructed by only modifying the Lennard-Jones parameters and the charges as well as the location of the charge sites were not modified. The parameterization was made only using experimental data [the $g_{\mathrm{OO}}(r), \rho$, and $\Delta H_{\mathrm{vap}}$ ] at $25^{\circ} \mathrm{C}$ and $1 \mathrm{~atm}$. Nevertheless, the model, like the original TIP5P model. is accurate over a range of temperatures and pressures. It is clear, at least, that when using Ewald sums, it would be better to use the TIP5P-E Lennard-Jones parameters than the original values. In addition, the Ewald and reaction field methods give very similar results and so the TIP5P-E parameters would be preferable to use with reaction field methods as well. It would be beneficial if the simulation community, including the developers of potentials, chose a method, or a set of compatible methods, for treating longranged electrostatics, in order to avoid problems like those illustrated in Fig. 2(A). The use of spherical cutoffs is among the least desirable methods and Ewald, which has both its critics $^{79}$ and its defenders, ${ }^{29}$ is perhaps the best choice.

\section{ACKNOWLEDGMENT}

Support from the National Science Foundation under Contract No. CHE-0213488 is gratefully acknowledged.

${ }^{1}$ A. Wallqvist and R. D. Mountain, in Reviews in Computational Chemistry, edited by K. B. Lipkowitz and D. B. Boyd (Wiley, Hoboken, New Jersey, 1999), pp. 183-247.

${ }^{2}$ M. W. Mahoney and W. L. Jorgensen, J. Chem. Phys. 112, 8910 (2000).

${ }^{3}$ H. A. Stern, F. Rittner, B. J. Berne, and R. A. Friesner, J. Chem. Phys. 115, 2237 (2001)

${ }^{4}$ M. W. Mahoney and W. L. Jorgensen, J. Chem. Phys. 114, 363 (2001).

${ }^{5}$ I. M. Svishchev, P. G. Kusalik, P. G. Wang, and R. J. Boyd, J. Chem. Phys. 105, 4742 (1996).

${ }^{6}$ B. Chen, J. Xing, and J. I. Siepmann, J. Phys. Chem. B 104, 2391 (2000).

${ }^{7}$ S. W. Rick, J. Chem. Phys. 114, 2276 (2001).

${ }^{8}$ Z. Bacsik, J. N. Canongia Lopes, M. F. Costa Gomes, G. Janscó, J. Mink, and A. A. H. Pádua, J. Chem. Phys. 116, 10816 (2002).

${ }^{9}$ S. Yoo and X. C. Zeng, J. Chem. Phys. 117, 9518 (2002).

${ }^{10}$ H. E. Stanley, S. V. Buldyrev, N. Giovambattista, E. La Nave, S. Mossa, A. Scala, F. Sciortino, F. W. Starr, and M. Yamada, J. Stat. Phys. 110, 1039 (2003)

${ }^{11}$ H. Nada and J. P. van der Eerden, J. Chem. Phys. 118, 7401 (2003).

${ }^{12}$ J. Bai, C. Su, R. D. Parra, X. Zeng, H. Tanaka, K. Koga, and J. Li, J. Chem. Phys. 118, 3913 (2003).

${ }^{13}$ R. Zangi and A. E. Mark, J. Chem. Phys. 119, 1694 (2003).

${ }^{14}$ R. B. Ayala and V. Tchijov, Can. J. Chem. 81, 11 (2003).

${ }^{15}$ C. Neito-Drago and J. B. Avalos, J. Chem. Phys. 118, 7954 (2003).

${ }^{16}$ Y. Lei, H. R. Li, H. H. Pan, and S. J. Han, J. Phys. Chem. A 107, 1574 (2003).

${ }^{17}$ C. Peltz, A. Baranyai, A. A. Chialvo, and P. T. Cummings, Mol. Simul. 29, 13 (2003).

${ }^{18}$ M. P. Allen and D. J. Tildesley, Computer Simulation of Liquids (Oxford University Press, Oxford, 1987).

${ }^{19}$ R. O. Watts, Mol. Phys. 4, 1069 (1974).

${ }^{20}$ C. Pangali, M. Rao, and B. J. Berne, Mol. Phys. 40, 661 (1980).

${ }^{21}$ T. A. Andrea, W. C. Swope, and H. C. Andersen, J. Chem. Phys. 79, 4576 (1983).

${ }^{22}$ C. L. Brooks III, B. M. Pettitt, and M. Karplus, J. Chem. Phys. 83, 5897 (1985).

${ }^{23}$ P. H. Hünenberger and W. F. van Gunsteren, J. Chem. Phys. 108, 6117 (1998).

${ }^{24}$ T. M. Nymand and P. Linse, J. Chem. Phys. 112, 6386 (2000).

${ }^{25} \mathrm{~S}$. W. Rick, in Simulation and Theory of Electrostatic Interactions in Solution, edited by L. R. Pratt and G. Hummer (American Institute of Physics, Melville, New York, 1999), pp. 114-126.

${ }^{26}$ S. E. Houstin and P. J. Rossky, J. Phys. Chem. 93, 7888 (1989).

${ }^{27}$ J. S. Bader and D. Chandler, J. Chem. Phys. 96, 6423 (1992).

${ }^{28}$ G. Hummer, D. M. Soumpasis, and M. Neumann, Mol. Phys. 81, 1155 (1993).

${ }^{29}$ G. Hummer, L. R. Pratt, and A. E. García, J. Phys. Chem. A 102, 7885 (1998).

${ }^{30}$ R. J. Loncharich and B. R. Brooks, Proteins: Struct., Funct., Genet. 6, 32 (1989).

${ }^{31}$ H. Schreiber and O. Steinhauser, Biochemistry 31, 5856 (1992).

${ }^{32}$ P. E. Smith and B. M. Pettitt, J. Chem. Phys. 95, 8430 (1991).

${ }^{33}$ D. M. York, T. A. Darden, and L. G. Pedersen, J. Chem. Phys. 99, 8345 (1993).

${ }^{34}$ T. E. Cheatham III, J. L. Miller, T. Fox, T. A. Darden, and P. A. Kollman, J. Am. Chem. Soc. 117, 4193 (1995)

${ }^{35}$ D. J. Adams, E. M. Adams, and G. J. Hills, Mol. Phys. 38, 387 (1979). 
${ }^{36} \mathrm{http} / / /$ amber.scripps.edu

${ }^{37} \mathrm{http}: / /$ www.scripps.edu/brooks/charmm_docs/charmm.html

${ }^{38}$ M. Lísal, J. Kolafa, and I. Nezbeda, J. Chem. Phys. 117, 8892 (2002).

${ }^{39}$ W. L. Jorgensen and J. D. Madura, Mol. Phys. 56, 1381 (1985).

${ }^{40}$ W. L. Jorgensen and C. Jenson, J. Comput. Chem. 19, 1179 (1998).

${ }^{41}$ S. L. Carnie and G. N. Patey, Mol. Phys. 47, 1129 (1982).

${ }^{42}$ M. Sprik, J. Chem. Phys. 95, 6762 (1991).

${ }^{43}$ H. C. Andersen, J. Chem. Phys. 72, 2384 (1980).

${ }^{44}$ G. Ciccotti and J. P. Ryckaert, Comput. Phys. Rep. 4, 345 (1986).

${ }^{45}$ G. J. Martyna, D. J. Tobias, and M. L. Klein, J. Chem. Phys. 101, 4177 (1994).

${ }^{46}$ S. Nosé, Mol. Phys. 52, 255 (1984).

${ }^{47}$ W. G. Hoover, Phys. Rev. A 31, 1695 (1985).

${ }^{48}$ D. Frenkel and B. Smit, Understanding Molecular Simulation: From Algorithms to Applications (Academic, San Diego, 1996).

${ }^{49}$ J. A. Barker and R. O. Watts, Mol. Phys. 26, 789 (1973).

${ }^{50}$ W. L. Jorgensen and C. Jensen, J. Comput. Chem. 19, 1179 (1998).

${ }^{51}$ R. W. Impey, P. A. Madden, and I. R. McDonald, Mol. Phys. 46, 513 (1982).

${ }^{52}$ G. Hura, J. M. Sorenson, R. M. Glaeser, and T. Head-Gordon, J. Chem. Phys. 113, 9140 (2000).

${ }^{53}$ T. Head-Gordon and G. Hura, Chem. Rev. (Washington, D.C.) 102, 2651 (2002).

${ }^{54}$ A. K. Soper, Chem. Phys. 258, 121 (2000).

${ }^{55}$ G. S. Kell, J. Chem. Eng. Data 20, 97 (1975).

${ }^{56}$ G. S. Kell, in Water-A Comprehensive Treatise, edited by F. Franks (Plenum, New York, 1972).

${ }^{57}$ N. E. Dorsey, Properties of Ordinary Water-Substance in All Its Phases: Water Vap or, Water, and all the Ices (Reinhold Publishing, New York, 1940).

${ }^{58}$ C. G. Malmberg and A. A. Maryott, J. Res. Natl. Bur. Stand. 56, 1 (1956).

${ }^{59}$ F. X. Prielmeier, E. W. Lang, R. J. Speedy, and H.-D. Lüdemann, Phys. Rev. Lett. 59, 1128 (1987).

${ }^{60}$ H. R. Pruppacher, J. Chem. Phys. 56, 101 (1972).

${ }^{61}$ W. L. Jorgensen, J. Chandrasekhar, J. D. Madura, R. W. Impey, and M. L. Klein, J. Chem. Phys. 79, 926 (1983).

${ }^{62}$ H. A. Stern, G. A. Kaminski, J. L. Banks, R. Zhou, B. J. Berne, and R. A. Friesner, J. Phys. Chem. B 103, 4730 (1999).
${ }^{63}$ H. J. C. Berendsen, J. P. M. Postma, W. F. van Gunsteren, A. DiNola, and J. R. Haak, J. Chem. Phys. 81, 3684 (1984).

${ }^{64}$ H. J. C. Berendsen, J. R. Grigera, and T. P. Straatsma, J. Phys. Chem. 91, 6269 (1987).

${ }^{65}$ K. Watanabe and M. L. Klein, Chem. Phys. 131, 157 (1989).

${ }^{66}$ J. Jonas, T. DeFries, and D. J. Wilbur, J. Chem. Phys. 65, 582 (1976).

${ }^{67}$ D. E. Smith and L. X. Dang, J. Chem. Phys. 100, 3757 (1994).

${ }^{68}$ T. Grindley and J. E. Lind, Jr., J. Chem. Phys. 54, 3983 (1971).

${ }^{69}$ J. Soetens, M. T. C. Martins Costa, and C. Millot, Mol. Phys. 94, 577 (1998).

${ }^{70}$ A. Chandra and T. Ichiye, J. Chem. Phys. 111, 2701 (1999).

${ }^{71}$ H. Yu, T. Hansson, and W. F. van Gunsteren, J. Chem. Phys. 118, 221 (2003).

${ }^{72}$ L. X. Dang, J. Chem. Phys. 97, 2659 (1992).

${ }^{73}$ S. W. Rick, S. J. Stuart, and B. J. Berne, J. Chem. Phys. 101, 6141 (1994).

${ }^{74}$ E. R. Batista, S. S. Xantheas, and H. Jónsson, J. Chem. Phys. 111, 6011 (1999).

${ }^{75}$ E. R. Batista, S. S. Xantheas, and H. Jónsson, J. Chem. Phys. 112, 3285 (2000).

${ }^{76}$ L. Delle Site, A. Alavi, and R. M. Lynden-Bell, Mol. Phys. 96, 1683 (1999).

${ }^{77}$ P. L. Silvestrelli and M. Parrinello, J. Chem. Phys. 111, 3572 (1999).

${ }^{78}$ For example, the model presented in Ref. 70 has no quadrupoles and a dielectric constant of 80 .

${ }^{79}$ P. H. Hünenberger and J. A. McCammon, J. Chem. Phys. 110, 1856 (1999).

${ }^{80}$ M. Neumann, J. Chem. Phys. 85, 1567 (1986).

${ }^{81}$ O. Matsuoka, E. Clementi, and M. Yoshimine, J. Chem. Phys. 64, 1351 (1976).

${ }^{82}$ M. Neumann, J. Chem. Phys. 82, 5663 (1985).

${ }^{83}$ H. J. C. Berendsen, J. P. M. Postma, W. F. van Gunsteren, and J. Hermans, in Intermolecular Forces, edited by B. Pullman (Reidel, Dordrecht, The Netherlands, 1981), pp. 331-342.

${ }^{84}$ T. N. Heinz, W. F. van Gunsteren, and P. H. Hünenberger, J. Chem. Phys. 115, $1125(2001)$.

${ }^{85}$ T. Dyke and J. Muenter, J. Chem. Phys. 59, 3125 (1973).

${ }^{86}$ J. Verhoeven and A. Dymanus, J. Chem. Phys. 52, 3222 (1970).

${ }^{87}$ M. Rami Ready and M. Berkowitz, Chem. Phys. Lett. 155, 173 (1989). 